\title{
Macular Degeneration and the Pharmacology of Lycium Berries
}

\author{
James Adams*, Yang Zhao, Rekha Sree and Gerson Navarrete \\ School of Pharmacy, Los Angeles, USA \\ *Corresponding author: James Adams, USC School of Pharmacy, Los Angeles, CA, USA
}

\section{ARTICLE INFO}

Received: 幽 November 20, 2019

Published: 慧 November 26, 2019

Citation: James Adams, Yang Zhao, Rekha Sree, Gerson Navarrete. Macular Degeneration and the Pharmacology of Lycium Berries. Biomed J Sci \& Tech Res 23(2)-2019. BJSTR. MS.ID.003868.

\section{ABSTRACT}

The pharmacology of carotenoids, flavonoids and polysaccharides is discussed in terms of their effects on the gut, immune cells and retinal functions. It is suggested that the use of goji berries may be superior to isolated, purified carotenoids and vitamins in the prevention of macular degeneration.

Keywords: Macular degeneration; zeaxanthin, Neovascularization; Flavonoids; Goji Berry Polysaccharides

Abbreviations: VEGF: Vascular Endothelial Growth Factor; BDNF: Brain Derived Neurotrophic Factor; TNF: Tumor Necrosis Factor; LBPs: Lycium Barbarum Polysaccharides

\section{Mini Review}

The retina is the light sensitive organ in the back of the eye. It is composed of 18 layers that contain neurons, Muller cells, ganglion cells, amacrine cells and other cells [1]. The retina also contains its own vasculature that supplies up to $25 \%$ of the oxygen and nutrients required by the retina [2]. The choroid, located between the retina and sclera, is the vascular layer of the eye and supplies the remaining $75 \%$ of the oxygen and nutrients needed by the retina [3]. The retinal macula and its central fovea are involved in sharp central vision and color perception. Retinal diseases decrease vision and can lead to blindness. Macular degeneration is an agerelated loss of central vision that is a cause of blindness in many people. Lifestyle, age and family history are prominent causes of macular degeneration [4]. Diet, smoking, high blood cholesterol, high blood pressure, lack of exercise and obesity are risk factors for the disease. Diabetic retinopathy is also a risk factor for macular degeneration [5]. The incidence of type 2 diabetes increases every year due to obesity [6], thereby increasing the risk of macular degeneration. Half of type 2 diabetes patients develop retinal pathology [7].

Late stage macular degeneration is described as a "wet" or "dry" form. The dry form is more common and progresses more slowly but can lead to wet macular degeneration. The wet form is

the leading cause of blindness in people over 60. The dry form is diagnosed by the presence of pigment clumps called drusen, small yellow deposits under the retina [8,9]. These drusen deposits may be the result of slow leakage from choroidal blood vessels. The wet form is diagnosed by visualizing extracellular fluid and blood in the macula that leaks out of blood vessels from the choroid. This visualization can be done with fluorescein dye, indocyanine green dye or optical coherence tomography $[8,9]$. Fluorescein dye can demonstrate the presence of leaking blood vessels and new blood vessels in the macula, called neovascularization. Inflammation of blood vessels in the macula with resultant leakage is a major cause of macular degeneration $[8,9]$. The retina attempts to repair itself following damage from drusen and neovascularization. Astrocytes and Muller cells form scars in the macula that repair the channels carved by extravasation of blood and fluids. This scarring can impair vision [8]. Treatment of wet macular degeneration involves antibodies against vascular endothelial growth factor (VEGF). Pegaptanib, ranibizumab, bevacizumab and aflibercept are the anti-VEGF antibodies used to inhibit neovascularization, fluid extravasation and restore vision in many patients $[8,9]$. These expensive drugs have greatly improved therapy for macular degeneration. However, they are also toxic and can increase strokes, hemorrhage and mortality [8]. 
There have been at least two clinical trials of vitamins and dietary supplements in the treatment of macular degeneration, AREDS and AREDS2. The AREDS study found that a combination of vitamin $C$, vitamin $E$, $\beta$-carotene, zinc and copper significantly decreased the risk of developing macular degeneration in patients with extensive or moderate drusen accumulation [10]. Due to the increased risk of lung cancer in patients receiving $\beta$-carotene, a new formulation containing lutein and zeaxanthin was tested in AREDS2 [11]. Lutein and zeaxanthin were found to be as good as $\beta$-carotene at preventing macular degeneration. Based on these trials, vitamin and mineral supplements are available that contain $10 \mathrm{mg}$ of lutein and $2 \mathrm{mg}$ of zeaxanthin in each tablet, as well as $500 \mathrm{mg}$ of Vitamin C, $400 \mathrm{U}$ of Vitamin E, $25 \mathrm{mg}$ of zinc and $2 \mathrm{mg}$ of copper. Inflammation of the retinal vasculature is important in macular degeneration $[7,8]$. This inflammation involves neutrophils and monocytes that infiltrate into the retina [12-14]. Neutrophils adhere to retinal endothelial cells and transmigrate into the macula due to the presence of integrin $\beta 1$ in early macular degeneration [12]. In diabetes, hyperglycemia and NADPH oxidase stimulate neutrophil extracellular trap formation on retinal endothelial cells [13]. This allows neutrophils to adhere and penetrate the macula, inducing inflammation. Monocytes are attracted into the macula by chemokines [14]. Once monocytes have penetrated the macula, they induce inflammation and neovascularization [14].

\section{Lycium Barbarum (Solanaceae)}

Goji berries from L. barbarum have been used in China for at least 2,000 years for aging and eye problems $[15,16]$. The normal dose of goji berries is 5-12g [16], usually steeped in Chrysanthemum tea. Each gram is about 3 goji berries. High levels of zeaxanthin are found in goji berries, about $1.87 \mathrm{mg} / \mathrm{g}$ of dried berries [16,17]. The zeaxanthin is present mostly as esters of palmitic acid. Goji berries also contain vitamin E and many other pharmacologically active compounds that may play roles in the prevention of macular degeneration. California has goji berries also. A recent publication found zeaxanthin and other active compounds in L. andersonii and L. cooperi from California [18]. California goji berries have been important medicines to California Indians for centuries. Goji berries have been used medically for macular degeneration, antioxidant effects, immune modulation, anti-inflammation and other effects [16]. The wide-ranging uses of goji berries suggests that several active compounds may be present that account for its clinical efficacy. Goji berries have been tested in a double blind; placebo controlled clinical trial (RCT) of retinitis pigmentosa [19]. Goji berries prevented cone cell death and macular thinning. An RCT of a goji berry extract in elderly patients found that macular characteristics improved [20]. Macular hypopigmentation and drusen accumulation were not as severe in the goji berry group. Clearly, additional RCTs are required to test the effects of goji berries in macular degeneration.

\section{Zeaxanthin}

Zeaxanthin is a xanthophyll carotenoid like lutein and cryptoxanthin. These are yellow pigments that absorb blue light. Lutein and zeaxanthin are isomers of each other, differing by the placement of a double bond. Natural sources include green, leafy vegetables like kale, chard and mustard greens. Administration of $15 \mathrm{~g}$ of goji berries daily for 28 days increased human plasma zeaxanthin levels 2.5-fold [16]. Zeaxanthin binds to high density lipoprotein and is transferred to the retina by a scavenger receptor [21]. This mechanism allows zeaxanthin to concentrate in the retina, especially the macula. Administration of zeaxanthin in a multi-vitamin and mineral form has been examined in clinical trials. A recent meta-analysis of all the clinical trials that found a zeaxanthin containing multi-vitamin mineral mixture may delay the progression of advanced macular degeneration [22]. This delay may be especially found in people exposed to more than normal oxidative stress, such as smokers, alcoholics and people who do not eat foods high in carotenoids. Some carotenoids, such as $\beta$-carotene are precursors for retinal, which is concentrated in rod cells and is necessary for vision.

Zeaxanthin has antioxidant effects that may protect retinal cells from the damaging effects of UV light [23]. It also increases human plasma brain derived neurotrophic factor (BDNF) and decreases human IL-1 $\beta$ [24]. Zeaxanthin may protect human retinal arteriolar endothelial cells thereby decreasing leaks into the retina [25]. BDNF supports the functions of retinal ganglion cells perhaps through a sigma-1 receptor or tropomyosin receptor kinase B mechanism $[26,27]$. IL-1 $\beta$ is an inflammatory protein that damages the retina by promoting chemokine production by Muller cells and the retinal pigmented epithelium [28]. IL-1 $\beta$ damages Muller cells by causing the nuclear accumulation of glyceraldehyde-3-phosphate dehydrogenase [29]. IL-1 $\beta$ induces forehead transcription factor 01 which damages human retinal microvascular endothelial cells [30], perhaps increasing the leakiness of retinal blood vessels. Zeaxanthin has several mechanisms of protection of the retina and the retinal microvasculature. It decreases IL-1 $1 \beta$ levels, thereby protecting Muller cells and preventing retinal scarring. It also protects endothelial cells, preventing neovascularization.

\section{Flavonoids}

The most abundant flavonoids in goji berries are quercetin and kaempferol [31]. The bioavailability of quercetin is low (about $2 \%$ ) but increases with daily consumption of fruits and vegetables rich in quercetin [32]. Retinal uptake of quercetin has not been reported. The glucuronide metabolite of quercetin is taken up into the human brain across the blood cerebrospinal fluid barrier [33]. This implies that the glucuronide of quercetin may also penetrate the retina. However, quercetin does not have to penetrate the retina in order to have beneficial effects on the retinal vasculature. It can 
exert its effects from the blood compartment. Quercetin treatments to diabetic rats decrease retinal microvascular pathology and leakiness [34]. IL-1 $\beta$ and tumor necrosis factor $\alpha$ (TNF $\alpha$ ) levels in the retinas of diabetic rats decrease due to quercetin [35]. Retinal thinning and apoptosis of diabetic rat retinal ganglion cells are inhibited by quercetin [35]. Quercetin has several effects in the vasculature involving signal transduction and phosphorylating enzymes [32]. These effects add up to enhanced vascular health in the presence of quercetin in the blood. Quercetin also has effects on the immune system [36]. It acts directly in the gut to affect the immune system, which is the site of maturation of many immune cells. Quercetin has several mechanisms of action including altering gene transcription, inhibiting phosphorylation enzymes, inhibiting matrix metalloproteinase, suppression of the activation and accumulation of immune cells [36].

Quercetin also acts on gut bacteria, such as Verrocomicrobia, and decreases the production of active lipid metabolites produced in the gut, such as lysophosphatidylcholine [37]. Several of these lipid metabolites may damage endothelial cells throughout the body. Quercetin inhibits microRNA production in gut dendritic cells [38]. Dendritic cells are involved in regulating the immune system, especially $\mathrm{T}$ cells and macrophages, that are involved in inflammation in the body. The bioavailability of kaempferol is low [39]. However, it penetrates the vasculature in enough quantities to alter signal transduction mechanisms in endothelial cells [39]. It especially inhibits phosphorylation enzymes that alter intracellular redox mechanisms. Kaempferol inhibits angiogenesis in human retinal endothelial cells by inhibiting VEGF expression [40]. This may inhibit neovascularization. Kaempferol is immunostimulatory, increases IgG antibody production and Th1, Th2 immune responses [41]. The stimulation of dendritic cells in the gut may be involved in this mechanism. Kaempferol also regulates gut bacteria and their production of potentially toxic, inflammatory metabolites [42].

These actions of kaempferol in the gut appear to be responsible for its anti-inflammatory activity. Rutin is found in goji berries and is a disaccharide of quercetin. The bioavailability of rutin is very low. However, rutin acts directly in the gut to inhibit the inflammatory effects of obesity on Paneth cells and inflammatory cytokine production [43]. Rutin also stimulates humoral and cellular immune responses [44]. Rutin administration to diabetic rats protects the retina by decreasing VEGF, retinal TNF $\alpha$ and retinal aldose reductase [45]. In this study, rutin was detected in the retina after systemic administration. Flavonoids have several mechanisms of action in the protection of the retina. Many of these mechanisms are based in the gut. It is not surprising that flavonoids have several mechanisms of action since human beings have been eating flavonoids for the entire 200,000 years of human existence. Humans have evolved eating flavonoids which may have influenced receptor evolution in the body.

\section{Lycium Barbarum Polysaccharides (LBPs)}

LBPs make up 5-8\% of the weight of dried goji berries [46]. These complex compounds have very low bioavailabilities and may act primarily in the gut. LBPs are immunostimulants by enhancing the production of B cells and chemokines [46]. T cells and macrophages are also stimulated by LBPs [46]. It is possible that stimulation of gut dendritic cells is involved in all these mechanisms [46]. LBPs modulate gut bacteria [47]. The effects of LBPs in the gut may result in immune and metabolic changes that protect the retina.

\section{Conclusion}

Focusing on zeaxanthin, lutein, minerals and vitamins in the prevention of macular degeneration provides effective therapy. However, goji berries with their arsenal of carotenoids, flavonoids and other compounds may provide superior therapy. Goji berry flavonoids and LBPs act in the gut to modulate the immune system, which may be beneficial in macular degeneration. Goji berries should be tested in more clinical trials. A gut retina axis is important in not only supplying nutrients to the retina, but also in regulating immune cells that affect the retina [48]. The use of goji berries may alter this gut retina axis in ways that decrease the progression of, or the risk of developing macular degeneration.

\section{References}

1. Cuenca N, Ortuño Lizarán I, Pinilla I (2018) Cellular Characterization of OCT and Outer Retinal Bands Using Specific Immunohistochemistry Markers and Clinical Implications. Ophthalmology 125(3): 407-422.

2. Campbell J, Zhang M, Hwang T, Bailey S, Wilson D, et al. (2017) Detailed vascular anatomy of the human retina by projection resolved optical coherence tomography angiography. Scientific Reports 7: 42201-42211.

3. Kur J, Newman E, Chan Ling T (2012) Cellular and physiological mechanisms underlying blood flow regulation in the retina and choroid in health and disease. Prog Retin Eye Res 31(5): 377-406.

4. Al Zamil WM, Yassin SA (2017) Recent developments in age-related macular degeneration: a review. Clin Interv Aging 12: 1313-1330.

5. He M, Chang F, Lin H, Wu J, Hsieh T, et al. (2018) The association between diabetes and age-related macular degeneration among the elderly in Taiwan. Diabetes Care 41(10): 2202-2211.

6. G Gruden, F Barutta, G Kunos, P Pacher (2016) Role of the endocannabinoid system in diabetes and diabetic complications. Brit J Pharmacol 173(7): 1116-1127.

7. Adams J, Parker K (2011) Extracellular and intracellular signaling. Royal Society of Chemistry, London.

8. Lim L, Mitchell P, Seddon J, Holz F, Wong T (2012) Ophthalmology 1. Age related macular degeneration. Lancet 379: 1728-1738.

9. Ong B, Ahfat F (2016) Age related macular degeneration. British Journal of Hospital Medicine 77: C18-C21.

10. (2001) Age-Related Eye Disease Study Research Group, A randomized, placebo-controlled, clinical trial of high-dose supplementation with vitamins $\mathrm{C}$ and $\mathrm{E}$, beta carotene and zinc for age-related macular degeneration and vision loss: AREDS report no. 8. Arch Ophthalmol 119(10): 1417-1436 
11. (2013) Age-Related Eye Disease Study 2 Research Group, Lutein + zeaxanthin and omega-3 fatty acids for age-related macular degeneration: the Age-Related Eye Disease Study 2 (AREDS2) randomized clinical trial. JAMA 309(19): 2005-2015.

12. Ghosh S, Padmanabhan A, Vaidya T, Watson AM, Bhutto IA, et al. (2019) Neutrophils homing into the retina trigger pathology in early age-related macular degeneration. Commun Biol 2: 348.

13. Wang L, Zhou X, Yin Y, Mai Y, Wang D, et al. (2019) Hyperglycemia Induces Neutrophil Extracellular Traps Formation Through an NADPH Oxidase-Dependent Pathway in Diabetic Retinopathy. Front Immunol 9: 3076.

14. Hagbi Levi S, Abraham M, Tiosano L, Rinsky B, Grunin M, et al. (2019) Promiscuous Chemokine Antagonist (BKT130) Suppresses LaserInduced Choroidal Neovascularization by Inhibition of Monocyte Recruitment. J Immunol Res p. 12.

15. Gao Y, Wei Y, Wang Y, Gao F, Chen Z (2017) Lycium Barbarum: A Traditional Chinese Herb and A Promising Anti-Aging Agent. Aging and Disease 8(6): 778-791.

16. Amagase H, Farnsworth N (2011) A review of botanical characteristics, Phytochemistry, clinical relevance in efficacy and safety of Lycium barbarum fruit (Goji). Food Research International 44(7): 1702-1717.

17. Niro S, Fratianni A, Panfili A, Falasca L, Cinquanta L, et al. (2017) Nutritional evaluation of fresh and dried goji berries cultivated in Italy. Ital J Food Sci 29(3): 398-408.

18. Navarrete G, Bracquemond A, Villasenor E, Wong M, Adams J (2019) Chemistry of California Lycium cooperi and Lycium andersonii. Eur Med Plants 29(3): 1-5.

19. Chan HH, Lam HI, Choi KY, Li SZ, Lakshmanan Y, et al. (2019) Delay of cone degeneration in retinitis pigmentosa using a 12-month treatment with Lycium barbarum supplement. J Ethnopharmacol 236: 336-344.

20. Bucheli P, Vidal K, Shen L, Gu Z, Zhang C, et al. (2011) Goji berry effects on macular characteristics and plasma antioxidant levels. Optom Vis Sci 88(2): 257-262.

21. Harrison E (2019) Mechanisms of Transport and Delivery of Vitamin A and Carotenoids to the Retinal Pigment Epithelium. Mol Nutr Food Res 63(15): e1801046.

22. Evans J, Lawrenson J (2017) Antioxidant vitamin and mineral supplements for slowing the progression of age-related macular degeneration. Cochrane Database Syst Rev 2017(7): CD000254.

23. Widjaja Adhi MAK, Ramkumar S, von Lintig J (2018) Protective role of carotenoids in the visual cycle. FASEB J.

24. Stringham NT, Holmes PV, Stringham JM (2019) Effects of macular xanthophyll supplementation on brain-derived neurotrophic factor, proinflammatory cytokines, and cognitive performance. Physiol Behav 211: 112650.

25. Kumari N, Cher J, Chua E, Hamzah H, Wong TY, et al. (2018) Association of serum lutein and zeaxanthin with quantitative measures of retinal vascular parameters. PLoS One 13(9): e0203868.

26. Mysona BA, Zhao J, Smith S, Bollinger KE (2018) Relationship between Sigma-1 receptor and BDNF in the visual system. Exp Eye Res 167: 2530 .

27. Liang Y, Yu YH, Yu HJ, Ma LS (2019) Effect of BDNF-TrKB pathway on apoptosis of retinal ganglion cells in glaucomatous animal model. Eur Rev Med Pharmacol Sci 23(9): 3561-3568.

28. Natoli R, Fernando N, Madigan M, Chu Tan JA, Valter K, et al. (2017) Microglia-derived IL-1 $\beta$ promotes chemokine expression by Müller cells and RPE in focal retinal degeneration. Mol Neurodegener 12(1): 31.

29. Yego EC, Vincent JA, Sarthy V, Busik JV, Mohr S (2009) Differential regulation of high glucose-induced glyceraldehyde-3-phosphate dehydrogenase nuclear accumulation in Müller cells by IL-1beta and IL6. Invest Ophthalmol Vis Sci 50(4): 1920-1928.
30. Wu L, Guo F, Wu Y, Wang Q Ma X, et al. (2017) The role of FoxO1 in interleukin-1 $\beta$-induced autostimulation in retina endothelial cells and retinas of diabetic rats. Microvasc Res 112: 93-100.

31. Qian D, Zhao Y, Yang G, Huang L (2017) Systematic Review of Chemical Constituents in the Genus Lycium (Solanaceae). Molecules 22(6).

32. Terao J (2017) Factors modulating bioavailability of quercetin-related flavonoids and the consequences of their vascular function. Biochem Pharmacol 139: 15-23.

33. Ishisaka A, Mukai R, Terao J, Shibata N, Kawai Y (2014) Specific localization of quercetin-3-0-glucuronide in human brain, Arch Biochem Biophys 557: 11-17.

34. Kumar B, Gupta S, Srinivasan B, Nag T, Srivastava S, et al. (2013) Quercetin protects retinal vascular degeneration through anti-angiogenic mechanisms in STZ-induced diabetic rats. Investigative Ophthalmology \& Visual Science 54: 5561.

35. Kumar B, Gupta SK, Nag TC, Srivastava S, Saxena R, et al. (2014) Retinal neuroprotective effects of quercetin in streptozotocin-induced diabetic rats. Exp Eye Res 125: 193-202.

36. Li Y, Yao J, Han C, Yang J, Tabassum Chaudhry M, et al. (2016) Quercetin, Inflammation and Immunity. Nutrients 8(3): 167.

37. Nie J, Zhang L, Zhao G, Du X (2019) Quercetin reduces atherosclerotic lesions by altering the gut microbiota and reducing atherogenic lipid metabolites. J Appl Microbiol 127(6): 1824-1834.

38. Galleggiante V, De Santis S, Liso M, Verna G, Sommella E, et al. (2019) Quercetin-Induced miR-369-3p Suppresses Chronic Inflammatory Response Targeting C/EBP- $\beta$. Mol Nutr Food Res 63(19): e1801390.

39. Devi KP, Malar DS, Nabavi SF, Sureda A, Xiao J, et al. (2015) Kaempferol and inflammation: From chemistry to medicine. Pharmacol Res 99:1-10.

40. Xu XH, Zhao C, Peng Q Xie P, Liu QH (2017) Kaempferol inhibited VEGF and PGF expression and in vitro angiogenesis of HRECs under diabeticlike environment. Braz J Med Biol Res 50(3): e5396.

41. Singh D, Tanwar H, Das S, Ganju L, Singh SB (2018) A novel in vivo adjuvant activity of kaempferol: enhanced Tbx-21, GATA-3 expression and peritoneal CD11c+MHCII+ dendritic cell infiltration. Immunopharmacol Immunotoxicol 40(3): 242-249.

42. Aa LX, Fei F, Qi Q Sun RB, Gu SH, et al. (2019) Rebalancing of the gut flora and microbial metabolism is responsible for the anti-arthritis effect of kaempferol. Acta Pharmacol Sin.

43. Guo X, Tang R, Yang S, Lu Y, Luo J, et al. (2018) Rutin and its combination with inulin attenuate gut dysbiosis, the inflammatory status and endoplasmic reticulum stress in Paneth cells of obese mice induced by high-fat diet. Front Microbiol 9: 2651

44. Ganeshpurkar A, Saluja AK (2017) Protective effect of rutin on humoral and cell mediated immunity in rat model. Chem Biol Interact 273: 154159.

45. Gupta SK, Sharma HP, Das U, Velpandian T, Saklani R (2019) Effect of rutin on retinal VEGF, TNF- $\alpha$, aldose reductase, and total antioxidant capacity in diabetic rats: molecular mechanism and ocular pharmacokinetics. Int Ophthalmol.

46. Jin M, Huang Q Zhao K, Shang P (2013) Biological activities and potential health benefit effects of polysaccharides isolated from Lycium barbarum L. International Journal of Biological Macromolecules 54: 16-23.

47. Ding Y, Yan Y, Chen D , Ran L, Mi J , et al. (2019) Modulating effects of polysaccharides from the fruits of Lycium barbarum on the immune response and gut microbiota in cyclophosphamide-treated mice. Food Funct 10(6): 3671-3683.

48. Rinninella E, Mele M, Merendino N, Cintoni M, Anselmi G, et al. (2018) The Role of Diet, Micronutrients and the Gut Microbiota in Age-Related Macular Degeneration: New Perspectives from the Gut-Retina Axis. Nutrients 10(11): 1677 


\section{ISSN: 2574-1241}

DOI: 10.26717/BJSTR.2019.23.003868

James Adams. Biomed J Sci \& Tech Res

(c) (P) This work is licensed under Creative

Submission Link: https://biomedres.us/submit-manuscript.php

\begin{tabular}{ll} 
BIOMEDICAL & Assets of Publishing with us \\
RESEARCHES & - Global archiving of articles \\
& - Immediate, unrestricted online access \\
\hline isSN: 2574-1241 & - Rigorous Peer Review Process \\
\end{tabular}

